\title{
Conventional Transarterial Chemoembolization (TACE) of a Hepatocellular Carcinoma with Aberrant Extrahepatic Blood Supply using Balloon Microcatheter for Flow Redirection
}

\section{Jain N, Chandra V, Contractor S and Kumar A*}

Department of Radiology, Rutgers New Jersey Medical School, USA

*Corresponding author: Abhishek Kumar, MD, Department of Radiology, Rutgers New Jersey Medical School, MSB F-506 185 South Orange Avenue, Newark, NJ, 07103, USA, Tel: 973-972-5188, Fax: 973-972-2307; Email: kumarab@njms.rutgers.edu

\section{Case Report \\ Volume 3 Issue 3}

Received Date: August 07, 2019

Published Date: August 27, 2019

DOI: $10.23880 /$ crij-16000152

\section{Abstract}

Hepatocellular carcinoma (HCC) is a malignant tumor that commonly affects cirrhotic patients. For tumors with a Barcelona Clinic Liver Cancer (BCLC) stage B, locoregional therapy is the mainstay of treatment. Transarterial chemoembolization (TACE) utilizes the hepatic arterial network in localizing chemotherapy to the HCC. 80\% of HCC cases are primarily supplied by the hepatic artery and most have extrahepatic collaterals from the inferior phrenic artery. This is the first reported case where the gastroduodenal artery (GDA) was the primary and sole blood supply to the HCC. The patient's levels for AST, ALT, hemoglobin, bilirubin, albumin, PT/INR, and platelets were all within normal range. The initial MRI showed a single exophytic tumor located on the caudate lobe. According to the BCLC staging system, the tumor was determined to be in the intermediate stage and TACE was considered to be the appropriate treatment. The right gastroepiploic artery was first occluded with detachable coils. Subsequently, a $150 \mathrm{~cm}$ straight $2.9 \mathrm{FrSniper}$ balloon microcatheter was introduced into the proximal GDA. TACE was performed using balloon occlusion to redirect blood flow away from the feeder arteries to the duodenum and into the blood supply to the tumor. One-month follow-up MRI scans showed reduction in the size of the HCC.

Keywords: Hepatocellular Carcinoma; Tumor; Transcatheter; Hemoglobin; Histopathology; Gastroepiploic Artery; Radiology

Abbreviations: HCC: Hepatocellular Carcinoma; BCLC: Barcelona Clinic Liver Cancer; TACE: Transarterial Chemoembolization; GDA: Gastroduodenal Artery; MRI:
Magnetic Resonance Imaging; TARE: Transarterial Radioembolization; EHC: Extrahepatic Collateral; DSA: Digital Subtraction Angiography; PPI: Proton Pump 


\section{Clinical Radiology \& Imaging Journal}

Inhibitors; B-TACE: Balloon-occluded Transcatheter Arterial Chemoembolization.

\section{Introduction}

Transcatheter directed locoregional therapies are currently the standard of care for patients with Barcelona Cancer Liver Clinic (BCLC) grade B (intermediate) hepatocellular carcinoma (HCC), as well as non BCLC stage $\mathrm{B}$ patients undergoing this treatment as a bridge therapy or as down staging [1].Several options exist for transcatheter directed therapy, such as transarterial chemoembolization (TACE) or transarterial radioembolization (TARE) [2,3]. In these interventions, chemotherapy or radiotherapy are directly injected into the hepatic arterial anatomy to selectively target tumor and spare non-diseased liver parenchyma. Hepatic arteries are the primary feeders in $80 \%$ of liver malignancies [4]. In patients with tumor sizes $>5 \mathrm{~cm}$, prior TACEs or surgeries, exophytic tumors, extrahepatic tumor infiltration, and/or tumor location at the bare area of the liver, extrahepatic collateral (EHC) arteries from nearby vasculature may provide additional supply in addition to a patent hepatic artery[5]. The right inferior phrenic artery contributes an additional blood supply to $70-83 \%$ of HCCs [5]. In rare cases, hepatic tumors in segments $V$ and VI that are close to the duodenum can have EHC from the gastroduodenal artery (GDA) and its branches [5]. However, no known cases have been reported where the GDA is the primary and sole blood supply to the HCC. Herein we present a case of an exophytic HCC in the caudate lobe with sole arterial supply via branches arising from the GDA.

\section{Clinical History}

A 71-year-old Caucasian male with a past medical history of hepatitis $\mathrm{C}$ infection and cirrhosis presented to a university hospital with jaundice, scleral icterus, abdominal pain, nausea, fatigue, weight loss, and chills. Baseline levels for AST, ALT, hemoglobin, bilirubin, albumin, PT/INR, and platelets were all within normal range. The patient's clinical presentation and lab results were consistent with a Child-Pugh Score A. Ascites and jaundice was not present.

\section{Imaging Review}

T1 weighted MRI of the abdomen displayed a single $6.0 \mathrm{~cm}$ exophytic tumor off the caudate lobe of the liver and arterial hyper enhancement with washout as shown in Figure 1. The patient was deemed to not be a good surgical candidate due to his portal hypertension. According to the BCLC staging system, the tumor was determined to be in the intermediate stage (B) and both TACE and TARE were considered as potential interventions.

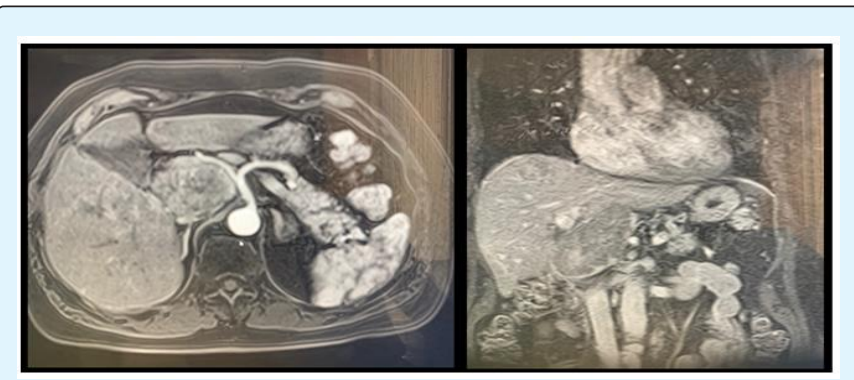

Figure 1: T1 weighted MR image of liver depicting $6 \mathrm{~cm}$ exophytic tumor originating from the caudate lobe of the liver.

\section{Treatment Options/Results}

In order to select the appropriate vessels for a TACE/TARE procedure, an initial angiogram was performed to visualize the vasculature supplying the HCC. Under ultrasound guidance, vascular access was obtained via the left radial artery using a TerumoGlidesheath5F Sarah Radial catheter. An initial arteriogram excluded any aberrant origins of the hepatic artery and a patent portal vein was visualized in the delayed phase. Celiac artery angiogram showed conventional left and right hepatic artery origins, but selective arteriograms of these vessels and their branches failed to demonstrate tumor enhancement. The GDA was the subselected with a $2.4 \mathrm{~F}$ microcatheter (Terumo Progreat). Angiogram displayed full blush of the tumor as shown in Figure 2.

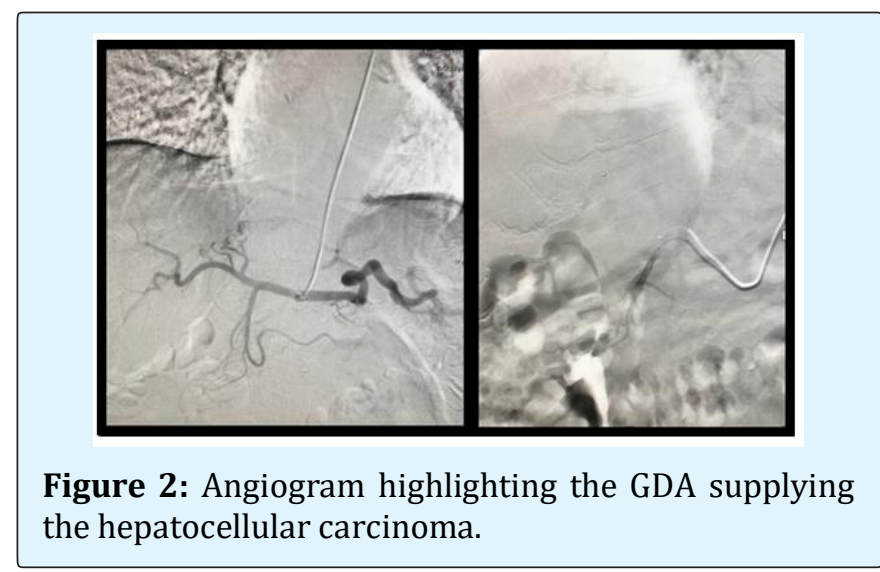

The GDA was confirmed to be the primary and sole blood supply of the HCC. A cone-beam CT was taken to 


\section{Clinical Radiology \& Imaging Journal}

identify additional organs supplied by the GDA, which showed enhancement of the tumor as well as the duodenum as shown in Figures 3\&4. From additional angiograms, it was determined that a branch of the GDA, the right gastroepiploic artery, supplied the duodenum.

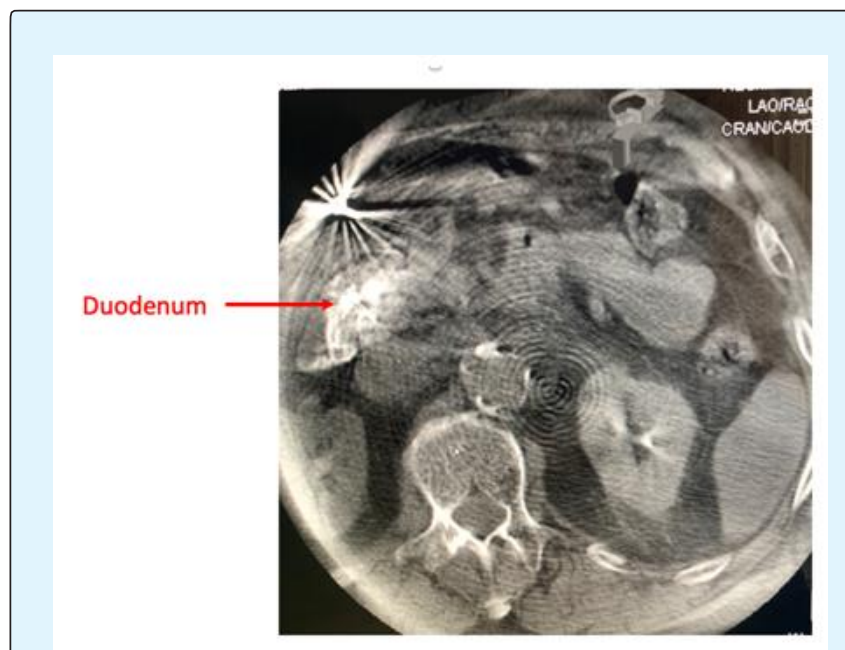

Figure 3: Cone beam CT depicting enhancement of the duodenum upon contrast injection into the GDA.

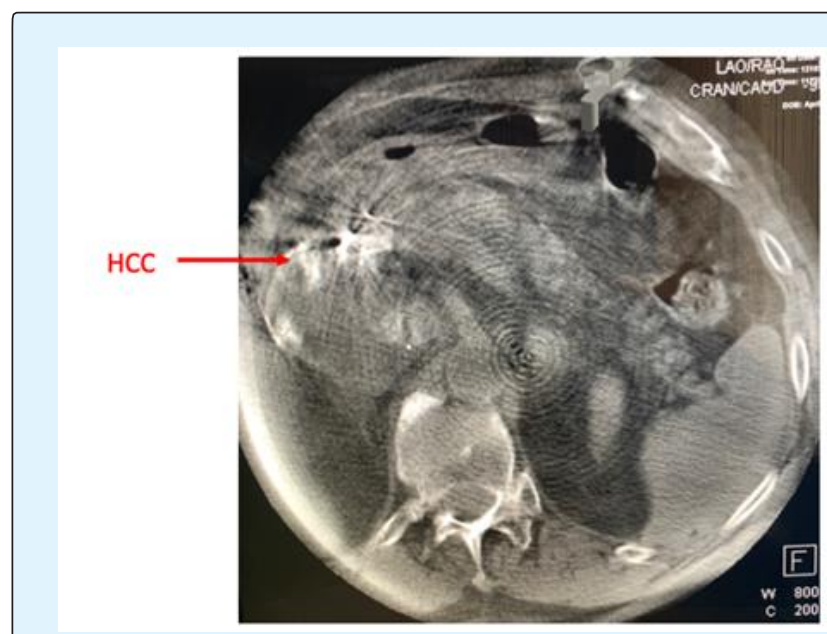

Figure 4: Cone beam CT depicting enhancement of the HCC upon contrast injection into the GDA.

The right gastroepiploic artery was first occluded with the $8 \mathrm{~mm}$ Concerto 3D Detachable Coil system. Subsequently, a $150 \mathrm{~cm}$ straight 2.9FrSniper balloon microcatheter was introduced into the proximal GDA. Balloon was inflated. Contrast injection demonstrated increased enhancement of the tumor and no flow to the duodenum.
cTACE was performed with 50mg doxorubicin emulsified in 8cc of ethiodol was performed until full enhancement of the HCC was seen on digital subtraction angiography (DSA) as seen in Figure 5.Patient placed on triple PPI therapy prophylactically to prevent ulcer.MRI evaluation one month later revealed $1 \mathrm{~cm}$ decrease in size and decreased enhancement of the tumor as shown in Figure 6.
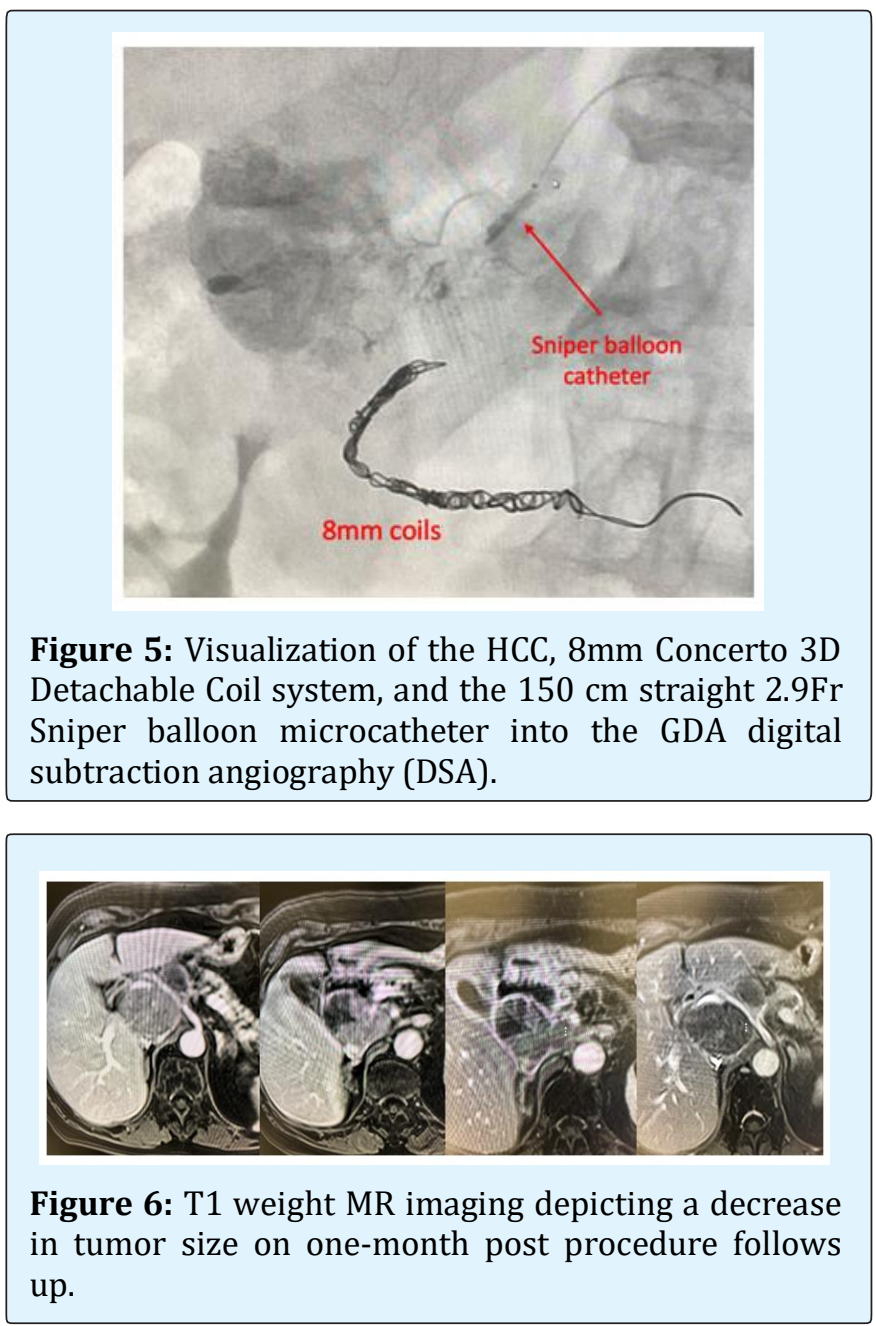

\section{Conclusion}

Many imaging modalities exist to properly diagnose HCC, magnetic resonance imaging (MRI) being the most sensitive. Once clinical suspicion and imaging suspect HCC, percutaneous liver biopsy is obtained to confirm the diagnosis [2]. Macroscopically, either a single large nodular mass or multiple nodules are visualized on gross examination. BCLC staging system guides treatment options for HCC [3]. This system accounts for the size and 


\section{Clinical Radiology \& Imaging Journal}

number of tumors in the liver and provides a reference on which intervention should be performed [2].

This patient's clinical presentation and histopathology suggested severe liver disease [2]. In patients with decompensated liver cirrhosis and HCC, the mortality rate is high [6]. Survival is usually dependent on a liver transplant. However, due to the scarcity of available livers, transplants at university centers in the US are considered only if the patient meets the Milan criteria [7]. The threshold criteria are as follows: the HCC is localized to one lesion $<5 \mathrm{~cm}$ or three lesions, each $<3 \mathrm{~cm}$, no extrahepatic manifestations, and no evidence of gross vascular invasion [8]. Since the patient's HCC measures to $6.0 \mathrm{~cm}$, the patient is not eligible for a transplant. The ultimate treatment goal for this patient was to reduce the tumor size to $<5 \mathrm{~cm}$ and downstage the patient for liver transplantation.

For single large lesions, the microcatheter is often positioned proximally in the feeding arteries. Since the GDA was the primary and sole feeder of the HCC, it was selected as the vessel of choice to deliver the chemotherapy. However, cone-beam CT confirmed that a branch of the GDA, the gastroepiploic artery, supplied the duodenum. If embolic particles accidently traveled down this branch and non-selectively embolized the gastroepiploic artery, GI bleeding and duodenum necrosis would subsequently ensue. Injecting too hard or fast beyond the endpoint of sluggish forward flow can cause reflux of embolic material into the duodenum. Non-target embolization is operator dependent. Confidence and comfort of the arterial anatomy is needed for prevention.

TACE and TARE have similar indications and were both considered for this patient. However, cTACE was deemed to be the better treatment option because of the potential of reflux. Reflux of the radioembolic particles into non-target regions is more catastrophic than reflux of chemoembolic particles [4]. Additionally, TARE consists of Yittrium-90radioembolic particles, which are much more difficult to control once inside the vessels [4]. Therefore, reflux was a relative contraindication of TARE embolization and cTACE was selected [4]. The Sniper balloon occlusion catheter was also used to regulate flow. Balloon-occluded transcatheter arterial chemoembolization (B-TACE) is an interventional approach that utilizes the infusion of lipiodol (an ethiodized oil) followed by gelatin particles under the occlusion of feeding arteries by a balloon catheter [9]. Using balloon occlusion, the Sniper microcatheter system created a low-pressure gradient at the proximal GDA, forcing blood flow towards the GDA and preventing reflux. While this hemodynamic gradient was created, the tip of the catheter ejected chemoembolic beads to selected portion of the GDA, leading to the accumulation in the HCC nodules.

One-month follow-up MRIs showed decrease in size of the HCC. To improve the probability for this patient to be considered for a liver transplant, the patient will return to the interventional radiology suite for additional TACE therapies.

\section{References}

1. Llovet JM, Fuster J, Bruix J, Barcelona-Clínic Liver Cancer Group (2004) The Barcelona approach: diagnosis, staging, and treatment of hepatocellular carcinoma. Liver Transpl 10(2):115-120.

2. Forner A, Llovet JM, Bruix J (2012) Hepatocellular carcinoma. The Lancet379 (9822):1245-1255.

3. Han K, Kim JH (2015) Transarterial Chemoembolization in Hepatocellular Carcinoma Treatment: Barcelona Clinic Liver Cancer Staging System. World J Gastroenterol 21(36):10327-10335.

4. WarhadpandeS, Lionberg A, Cooper KJ (2019) Pocketbook of Clinical IR A Concise Guide to Interventional Radiology. Thieme Medical Publishers.

5. Moustafa AS, Abdel Aal AK, Ertel N, Saad N, DuBay D, et al. (2017) Chemoembolization of Hepatocellular Carcinoma with Extrahepatic Collateral Blood Supply: Anatomic and Technical Considerations. Radio Graphics 37(3): 963-977.

6. Tapper EB, Parikh ND (2018) Mortality Due to Cirrhosis and Liver Cancer in the United States, 19992016: observational Study. BMJ: 362.

7. Zhijun Z (2016) Milan Criteria and Its Expansions in Liver Transplantation for Hepatocellular Carcinoma. Hepatobiliary SurgNutr 5(6): 498-502.

8. Kim HC, Chung JW, Lee W, Jae HJ, Park JH (2005) Recognizing Extrahepatic Collateral Vessels That Supply Hepatocellular Carcinoma to Avoid Complications of Transcatheter Arterial Chemoembolization. Radiographics 25(1): 25-39.

9. Hatanaka T, Arai H, Kakizaki S (2018) Balloonoccluded transcatheter arterial chemoembolization for hepatocellular carcinoma.World J Hepatol10(7): 485-495. 